\title{
Editorial
}

\section{Machine Intelligence in Signal Sensing, Processing, and Recognition}

\author{
Lei Zhang, ${ }^{1}$ Sunil Kr. Jha, ${ }^{2}$ Zhixin Yang, ${ }^{3}$ Zhenbing Zhao, ${ }^{4}$ and Bhupendra Nath Tiwari \\ ${ }^{1}$ College of Communication Engineering, Chongqing University, Chongqing, China \\ ${ }^{2}$ Chair of Mathematics, IT Fundamentals and Education Technologies Applications, University of Information Technology and \\ Management in Rzeszow, Rzeszow, Poland \\ ${ }^{3}$ Faculty of Science and Technology, University of Macau, Taipa, Macau \\ ${ }^{4}$ North China Electric Power University, Baoding, China \\ ${ }^{5}$ INFN-Laboratori Nazionali di Frascati, Rome, Italy \\ Correspondence should be addressed to Lei Zhang; leizhang@cqu.edu.cn
}

Received 25 July 2017; Accepted 25 July 2017; Published 6 September 2017

Copyright (C) 2017 Lei Zhang et al. This is an open access article distributed under the Creative Commons Attribution License, which permits unrestricted use, distribution, and reproduction in any medium, provided the original work is properly cited.

In recent years, machine intelligence has become a wellestablished research area and attracted a number of researchers in many science and engineering fields, for example, robotics, artificial intelligence, big data, IoT, and smart things. Many communities such as signal processing, intelligent sensing, image/video processing, computer vision, machine learning, deep learning, transfer learning, extreme learning machine, and representational learning are playing important role in machine intelligence [1-4]. The common goal is to develop new techniques and algorithms for making more intelligent things that can provide more comfortable living conditions for the world.

In general, conventional machine intelligence includes three phases, such as signal sensing, signal processing, and signal recognition, which are also termed as low-level acquisition, middle-level representation, and high-level analysis. Beyond the conventional intelligence, artificial intelligence also integrates the elements of rich big data, high computational ability, efficient learning algorithms, Internet, and chips. Learning algorithms play a critical role in AI developments. A number of researchers from different fields, such as computer vision, natural language processing, remote sensing, medical diagnosis, smart grid, and system control, have been attracted by the popular deep learning algorithms. Further research on signal sensing, processing, and recognition can be investigated and proposed for providing more perspective in machine intelligence.

In this special issue, novel treatments and applications of signal processing and machine learning algorithms have been explored in different fields, including speaker recognition, environmental data analysis, remote sensing data modeling, fault diagnosis, and computer vision. Algorithms such as extreme learning machine, Bayesian inference, Bayesian network, least square regression, and wavelets have been exploited. This special issue provides readers with new insight about signal based sensing, processing, and recognition in machine intelligence topics, which are highly interesting and scientifically valid.

\section{Lei Zhang \\ Sunil Kr. Jha \\ Zhixin Yang \\ Zhenbing Zhao \\ Bhupendra Nath Tiwari}

\section{References}

[1] Y. LeCun, Y. Bengio, and G. Hinton, "Deep learning," Nature, vol. 521, no. 7553, pp. 436-444, 2015.

[2] G.-B. Huang, "What are extreme learning machines? Filling the gap between Frank Rosenblatt's dream and John Von 
Neumann's puzzle," Cognitive Computation, vol. 7, no. 3, pp. 263-278, 2015.

[3] L. Zhang, W. Zuo, and D. Zhang, "LSDT: latent sparse domain transfer learning for visual adaptation," IEEE Transactions on Image Processing, vol. 25, no. 3, pp. 1177-1191, 2016.

[4] Y. Bengio, A. Courville, and P. Vincent, "Representation learning: a review and new perspectives," IEEE Transactions on Pattern Analysis and Machine Intelligence, vol. 35, no. 8, pp. 1798-1828, 2013. 


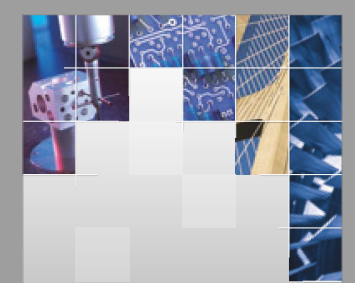

\section{Enfincering}
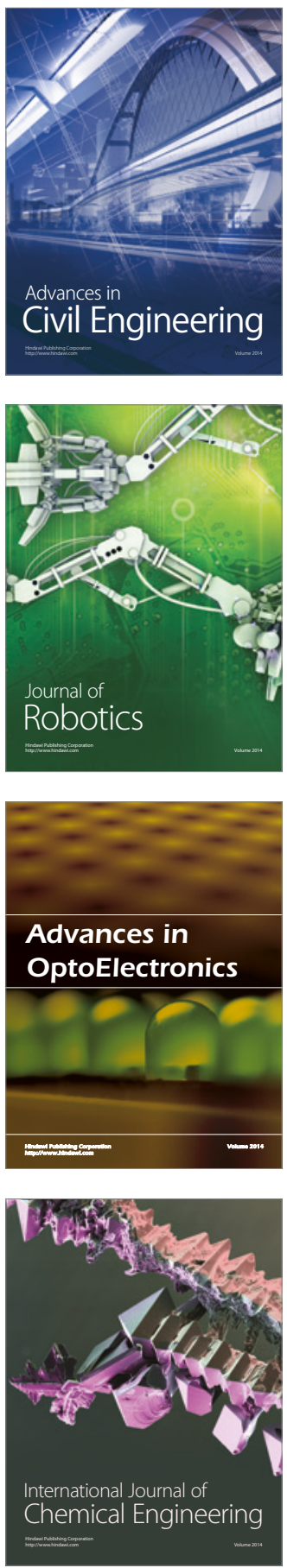

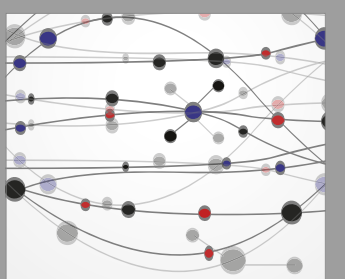

The Scientific World Journal

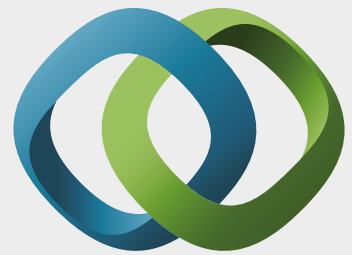

\section{Hindawi}

Submit your manuscripts at

https://www.hindawi.com
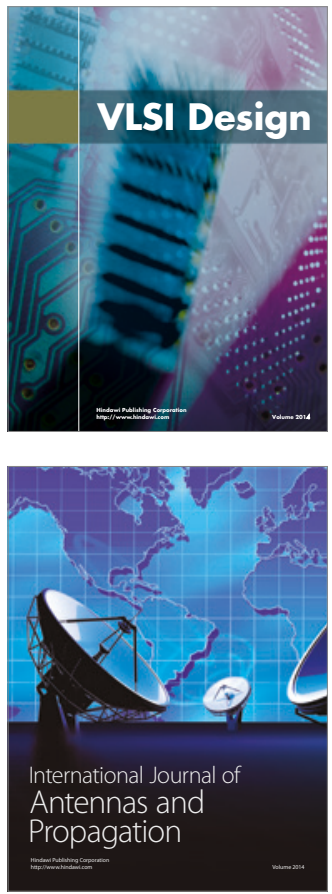

\section{Rotating}

Machinery
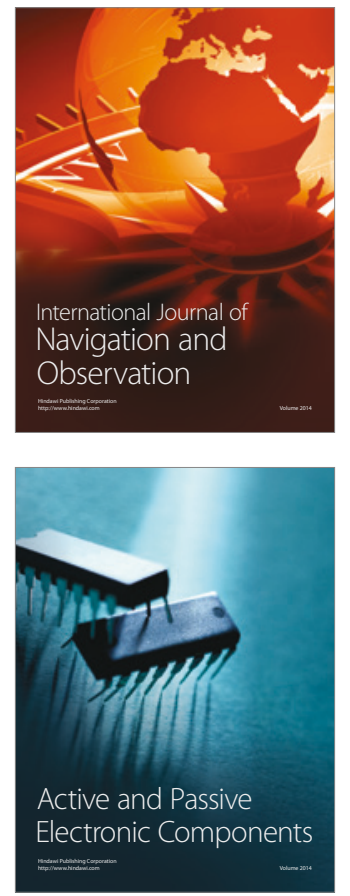
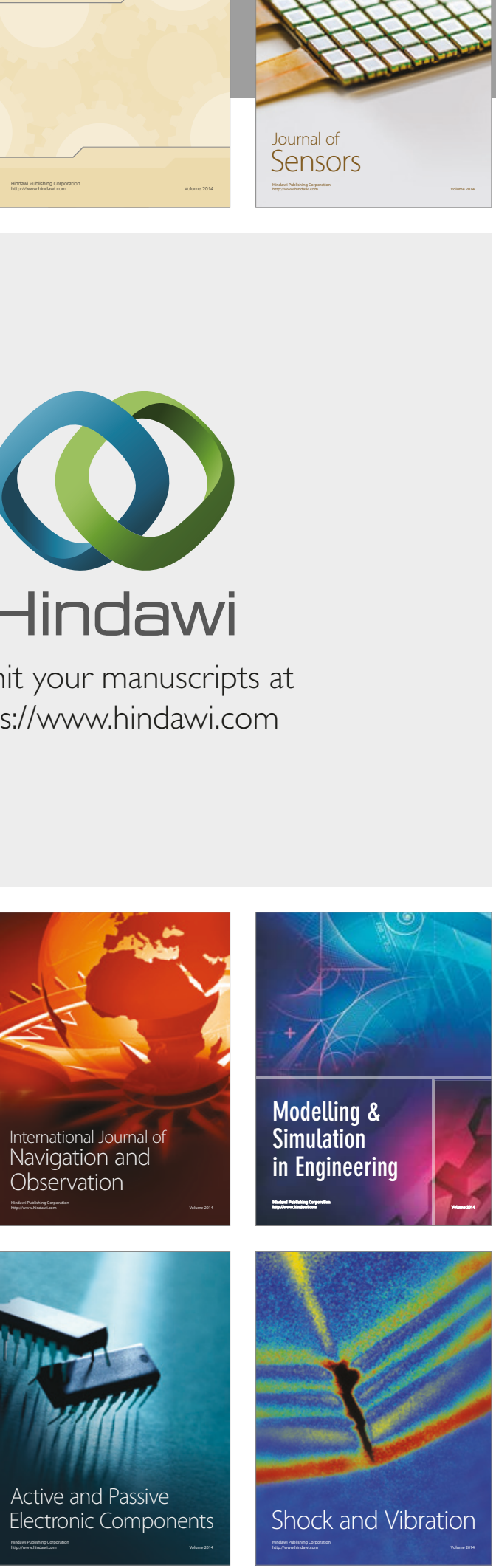
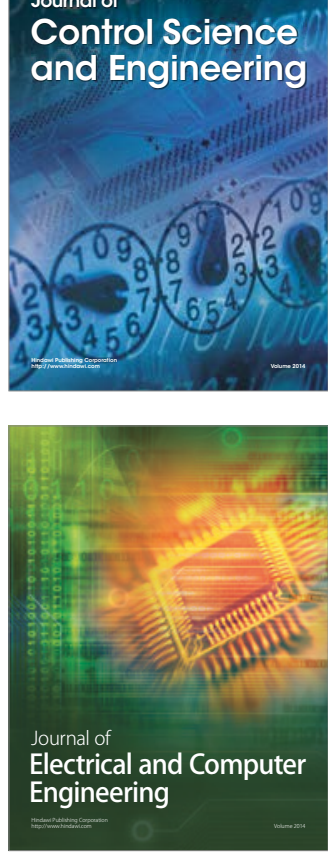

Distributed

Journal of

Control Science

and Engineering
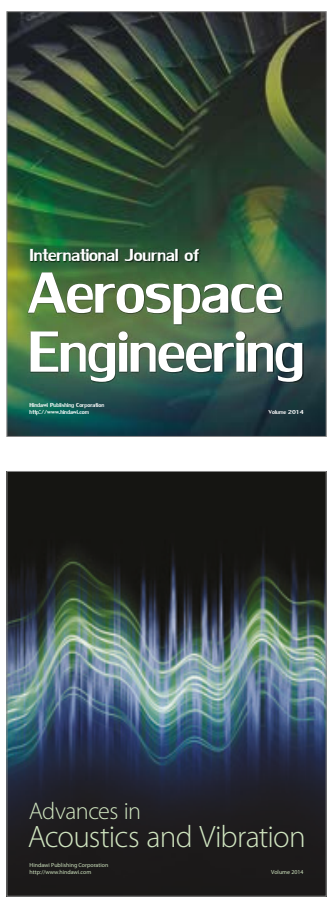

Sensor Networks 